\title{
Manejo anestesico perioperatorio de la corrección de pectus excavatum con técnica de pectus up. Reporte de caso
}

\section{Perioperatory anesthetic management of pectus excavatum correction with pectus up technique. Case report}

\author{
Alexander Trujillo MD, MSc. ${ }^{1,2, *}$, Ramiro Moreno ${ }^{1}$ \\ Facultad de Ciencias para la Salud, Universidad de Caldas, Manizales, Colombia. \\ 2 Programa de Medicina, Universidad de Manizales, Colombia.
}

Conflicto de intereses: Los autores declaran no tener conflicto de interés.

Financiamiento: El manuscrito fue realizado con recursos propios de los autores.

Confidencialidad de los datos: Los autores declaran que han seguido los protocolos de su centro de trabajo sobre publicación de datos de pacientes.

Derecho a la privacidad: Los autores declaran que este artículo protege la privacidad del paciente. Se obtuvo consentimiento informado por escrito para la publicación del caso.

Fecha de recepción: 15 de mayo de 2021 / Fecha de aceptación: 15 de junio de 2021

\begin{abstract}
Introduction: Pectus excavatum is the most common congenital anomaly of the thorax. Various surgical procedures have been proposed for its correction. Recently, the pectus up surgery or taulinoplasty has been introduced as a good therapeutic option. However, its perioperative anesthetic management has not been described. Case presentation: A 13-year-old patient with severe pectus excavatum underwent correction with pectus up surgery. The anesthetic management was performed with balanced anesthesia with Sevoflurane and Remifentanil. The analgesic strategy consisted of ultrasound-guided bilateral transverse thoracic muscle fascia block plus multimodal analgesia. The patient was discharged on the third day without complications. Conclusions: Pectus up surgery is a surgical option for the correction of pectus excavatum. Multimodal treatment with intravenous analgesia and ultrasound-guided bilateral transverse thoracic muscle fascia block are a good option for postoperative pain control.
\end{abstract}

Key words: Pectus excavatum, taulinoplasty.

\section{RESUMEN}

Introducción: El pectus excavatum es la anomalía congénita del tórax más frecuente. Diversos procedimientos quirúrgicos han sido propuestos para su corrección. Recientemente la cirugía de pectus up o taulinoplastia se ha introducido como una buena opción terapéutica sin que el manejo anestésico perioperatorio haya sido descrito. Presentación del caso: Un paciente de 13 años de edad con pectus excavatum severo, fue sometido a corrección con cirugía de pectus up. El manejo anestésico se realizó con anestesia balanceada con Sevoflurano y Remifentanil. La estrategia analgésica consistió en el bloqueo de la fascia del músculo transverso toráxico bilateral ecoguíado más analgesia multimodal. El paciente fue dado de alta al tercer día sin complicaciones. Conclusiones: La cirugía de pectus up es una opción quirúrgica para la corrección del pectus excavatum. El tratamiento multimodal con analgesia i.v. y el bloqueo de la fascia del músculo transverso torácico bilateral ecoguiado son una buena opción para el control del dolor posoperatorio.

Palabras clave: Pectus excavatum, taulinoplastia. 


\section{Introducción}

pectus excavatum (PE) es la anomalía congénita del tórax más frecuente. La etiología es múltiple, siendo idiopática en el $50 \%$ de los casos, hereditaria en el $40 \%$ y debida a alteraciones del colágeno como en el síndrome de Marfán y enfermedad de Ehler-Danlons en el $4 \%$ y $1 \%$ de los casos, respectivamente. El defecto torácico se acentúa con el crecimiento, especialmente durante la pubertad, cuando la circunferencia torácica aumenta el 50\%.

Existen diversos procedimientos quirúrgicos para la corrección del PE. Recientemente, la cirugía de pectus up también denominada taulinoplastia ha aumentado las opciones terapéuticas. Sin embargo, el manejo anestésico perioperatorio no ha sido descrito.

El propósito de este reporte de caso es compartir nuestras experiencias en el manejo anestésico perioperatorio de un paciente intervenido en el Hospital Infantil Rafael Henao Toro de la Cruz Roja de Manizales, Colombia.

\section{Presentación del caso}

Paciente masculino de 13 años de edad, caucásico, estudiante de bachillerato, procedente del área urbana de Manizales, Colombia, asistió a consulta preanestésica programado para corrección de PE con técnica de pectus up (taulinoplastia). El defecto de la pared torácica había sido identificado después del nacimiento y en controles periódicos se documentó el aumentando progresivo y asimétrico de la deformidad torácica con el crecimiento. Durante la primera infancia, fue diagnosticado con asma intermitente, sin recurrencia de síntomas para el momento de la cirugía. En tratamiento con salbutamol inhalado a demanda y montelukast $5 \mathrm{mg} /$ día vía oral. Sin antecedentes alérgicos, tóxicos ni quirúrgicos.

En el examen físico peso: 57 kg, estatura: $179 \mathrm{~cm}$, gran deformidad torácica con depresión del esternón, asimetría en los hemitórax y en la altura de las escápulas, asociado a una notoria escoliosis dorso lumbar. La auscultación cardiopulmonar fue normal. No se encontraron predictores de vía aérea difícil. El resto del examen físico fue normal (Figura 1).

La tomografía axial computarizada del tórax evidenció un
PE severo (índice de Haller de 5,2), (Figura 2A), el ecocardiograma fue normal; la espirometría informó broncoconstricción leve inducida por el ejercicio. La analítica sanguínea reportó: hemoglobina: $16,8 \mathrm{~g} / \mathrm{dL}$, hematocrito: $52 \%$, leucocitos: 5.240 $10^{3} / \mu \mathrm{L}$, plaquetas: $197.00010^{3} / \mu \mathrm{L}$. Las pérdidas permisibles de sangre calculadas fueron de: $1.304 \mathrm{~mL}$. Se reservó cama en cuidado intensivo y 2 unidades de concentrado globular. El día previo se realizó prueba de hipersensibilidad cutánea al material de la placa que fue negativa.

Se realizó monitoria básica, inducción anestésica venosa con remifentanil $2 \mu \mathrm{g} / \mathrm{kg}$ y propofol $2 \mathrm{mg} / \mathrm{kg}$, vía aérea manejada con tubo orotraqueal monolumen No 7.0 con neumotaponador y ventilación controlada por volumen con volumen tidal

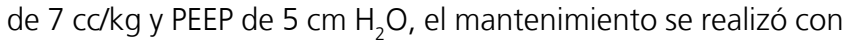
anestesia balanceada remifentanil $0,2, \mu \mathrm{g} / \mathrm{Kg} / \mathrm{min}$ y sevoflorane exhalado $2 \%$ en oxígeno al $60 \%$. Antes de la incisión quirúrgica, se realizó bloqueo guiado por ultrasonido del plano de la fascia del músculo transverso torácico de manera bilateral con $20 \mathrm{~mL}$ de bupivacaína al 0,25\% con epinefrina. El intraoperatorio transcurrió con estabilidad hemodinámica, mínimas pérdidas sanguíneas y sin complicaciones (Figura 2B). El manejo analgésico consistió en dipirona $2 \mathrm{gr}$, hidromorfona $800 \mu \mathrm{g}$ y ketamina $20 \mathrm{mg}$ i.v. El tiempo quirúrgico fue de 150 minutos.

El posoperatorio se realizó en hospitalización. Se instauró manejo analgésico con acetaminofén 500 mg cada 6 h vía oral, nimesulide 100 mg cada 12 h v.o. y dipirona 1,5 g cada 6 h i.v. Se ordenó hidromorfona como rescate en caso de dolor $\geq 5 / 10$, sin haber requerido dosis alguna. Evolucionó satisfactoriamente dándose alta al tercer día con acetaminofén 500 mg cada 6 h y nimesulide $100 \mathrm{mg}$ cada $12 \mathrm{~h}$. Al séptimo día se realizó el primer control posoperatorio donde manifestó dolor leve en el sitio quirúrgico.

\section{Discusión}

El PE es la anomalía congénita del tórax más común, afectando a los hombres 5 veces más que a las mujeres. El PE es el resultado de una fusión inadecuada de las costillas con el esternón durante el desarrollo embriológico. El signo de presentación cardinal es la depresión torácica que puede ser evidente

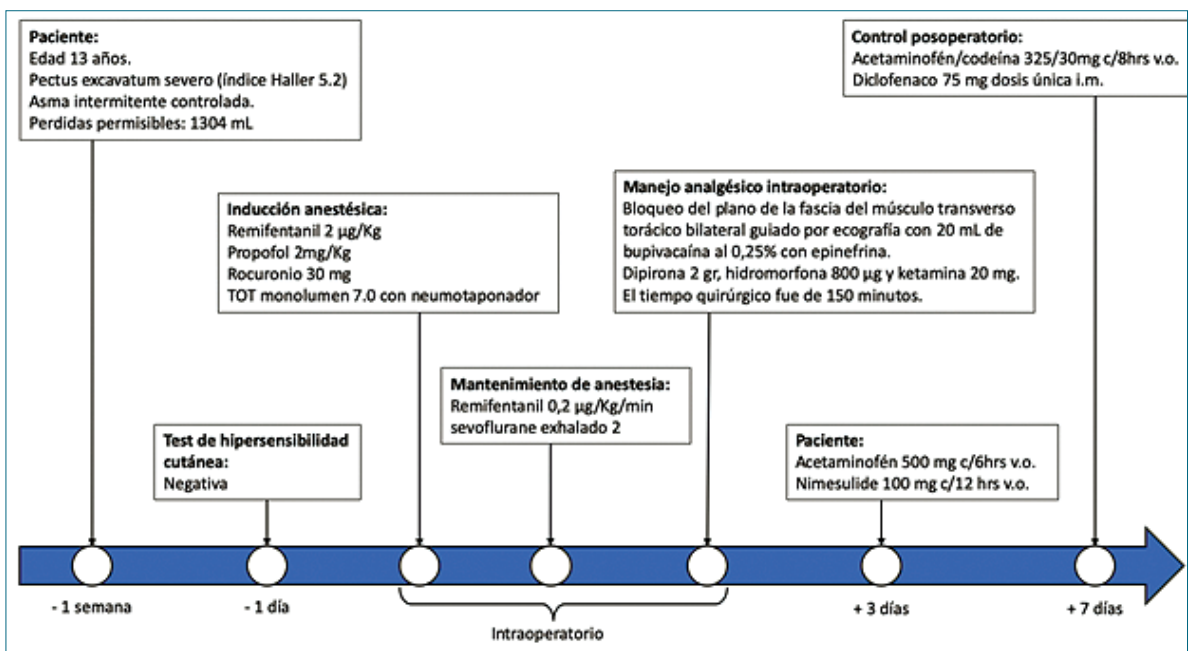

Figura 1. Línea de tiempo de los eventos clínicos. 


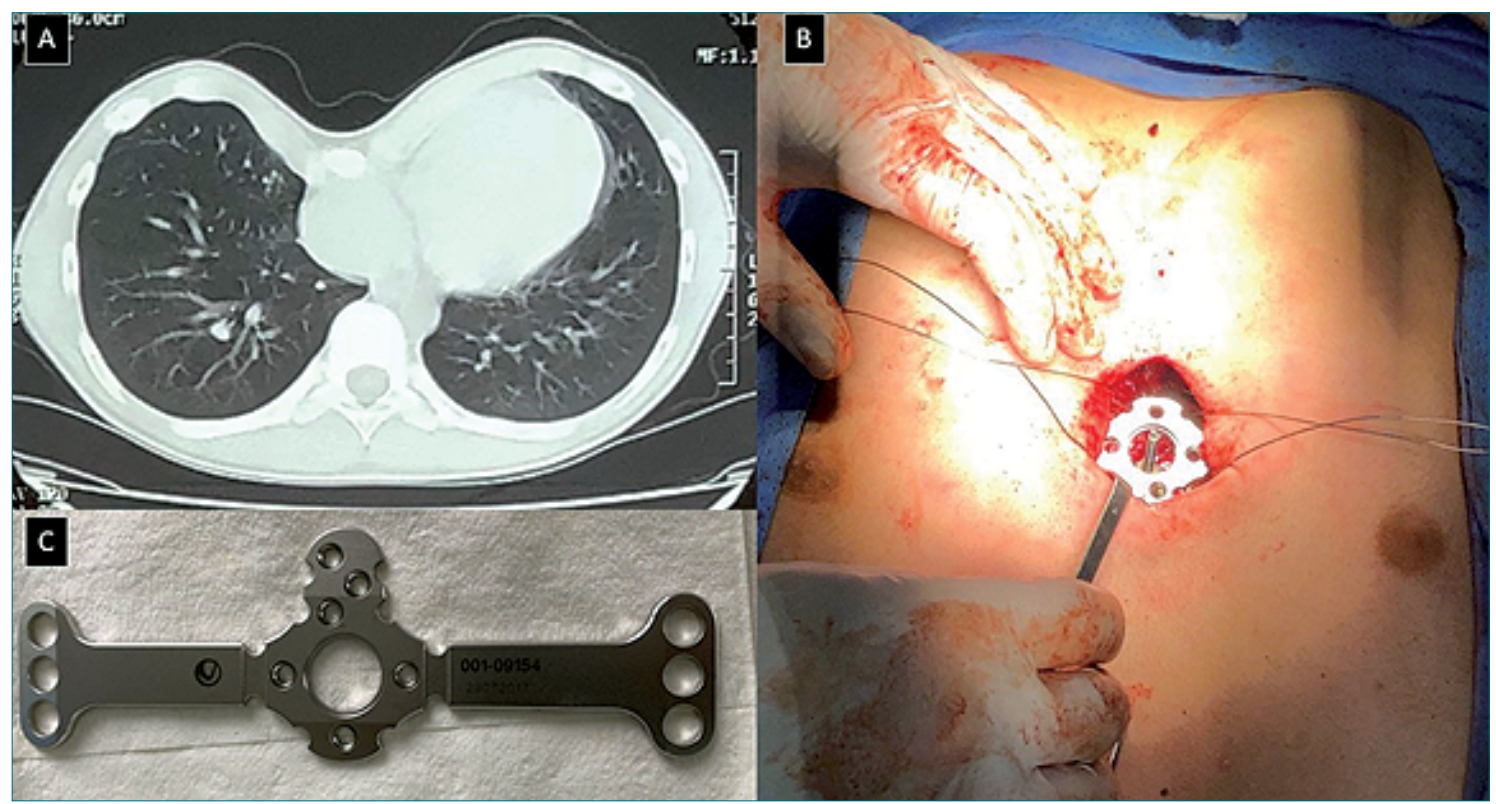

Figura 2. A: Tomografía Axial Computarizada, índice de Haller: 5,2; B: Placa y sistema de tracción para pectus up puesta sobre el esternón; C: Placa de pectus up.

al nacimiento y acentuarse en la adolescencia. Los pacientes pueden quejarse de dolor torácico, disnea de esfuerzos y tener reducida su capacidad para realizar ejercicio. Además, el PE puede generar alteraciones de la autoestima llevando al aislamiento social, depresión e incluso ideación suicida. La evaluación preoperatoria incluye la medición tomográfica de la deformidad torácica a través del índice de Haller, considerándose un defecto severo si es mayor a 3,2. Además, debe realizarse una evaluación de la función cardiopulmonar, incluidas las arritmias y las pruebas de función pulmonar[1].

El paciente presentaba un índice de Haller de 5,2 clasificado como severo por lo que se consideró necesaria la corrección quirúrgica. Tradicionalmente, este tipo de defectos han sido corregidos por vía abierta o técnica de Ravitch, en la que se realiza resección de los cartílagos costales deformados, separación del xifoides del esternón y osteotomía esternal transversal para desplazar el esternón anteriormente[2] y técnicas menos invasivas como el procedimiento de Nuss mediante la inserción guiada por toracoscopia de una barra de titanio debajo del esternón fijada lateralmente a las costillas. Un metaanálisis que incluyó 1.432 pacientes pediátricos, no encontró diferencias entre ambas técnicas en la frecuencia de complicaciones totales y específicas (reintervenciones, infección, neumonía, neumotórax o hemitórax)[3]. Otro metaanálisis que incluyó 1.731 pacientes pediátricos, encontró diferencias significativas en el tiempo quirúrgico entre ambas técnicas (diferencia media estándar acumulada: $-2,83$, IC95\%: -3,76 a -1,90, p < 0,001) y sangrado intraoperatorio (diferencia media estándar acumulada: -1,68, IC95\%: -2,28 a -1,09, p < 0,001)[4]. En estas técnicas, el dolor posoperatorio es un obstáculo frecuente para el alta temprana, reportándose promedios de hospitalización cercanos a los 7 días[1]. Además, el retiro posterior de la barra puede ser dificultosa y generar complicaciones.

Recientemente, la técnica del pectus up o taulinoplastia ha sido propuesta como una buena alternativa para corrección del
$P E$, ya que evita la pleura y el mediastino y genera menor dolor posoperatorio, acortando la estancia hospitalaria. Esta técnica consiste en la fijación de una placa extratorácica con un sistema de tracción en la cara anterior del esternon[5] (Figura 2C). Por tratarse de una técnica relativamente nueva, poco se ha informado sobre las mejores estrategias de analgesia posoperatoria[6]. En nuestro hospital existe un protocolo de analgesia multimodal para PE corregidas con barras de Nuss, consistente en analgesia peridural torácica con infusión continua de bupivacaína 0,1\% y morfina, dexmedetomidina en infusión continua y dipirona i.v, siendo manejado el paciente en UCI durante los 2 primeros días. En este caso, modificamos nuestro protocolo al considerar que el procedimiento era menos invasivo, por lo que se realizó un bloqueo de la fascia del músculo transverso torácico de manera bilateral guiado por ultrasonido. Este bloqueo se realiza inyectando $20 \mathrm{~mL}$ de bupivacaina al 0,25\% con epinefrina entre el músculo torácico transverso y el musculo intercostal interno a cada lado del esternón para bloquear las ramas anteriores de los nervios intercosales $2^{\circ}$ a $6^{\circ}$. Es muy importante identificar la arteria mamaria interna, para evitar la inyección intravascular. Este bloqueo ha mostrado eficacia en esternotomías[7],[8] y cirugía de mama, sin encontrarse reportes en pectus excavatum. Nuestra experiencia demuestra un adecuado control del dolor con este bloqueo, permaneciendo en una EVA por debajo de 5, sin requerir rescates con opioides, a pesar de no tener analgesia peridural ni dexmedetomidina. Por ello, pudo ser manejado en hospitalización y ser dado de alta al tercer día con analgésicos orales.

\section{Conclusión}

La cirugía de pectus up es una opción quirúrgica para la corrección de PE. El tratamiento multimodal con dipirona, acetaminofén y el bloqueo de la fascia del músculo transverso to- 
rácico bilateral ecoguiado son una buena opción para el control del dolor posoperatorio.

\section{Referencias}

1. Abdullah F, Harris J. Pectus Excavatum: More Than a Matter of Aesthetics. Pediatr Ann. 2016 Nov 1;45(11):e403-e406. https:// doi.org/10.3928/19382359-20161007-01

2. Antonoff MB, Erickson AE, Hess DJ, Acton RD, Saltzman DA. When patients choose: comparison of Nuss, Ravitch, and Leonard procedures for primary repair of pectus excavatum. J Pediatr Surg. 2009 Jun;44(6):1113-8; discussion 118-9. https://doi. org/10.1016/j.jpedsurg.2009.02.017

3. Kanagaratnam A, Phan S, Tchantchaleishvili V, Phan K. Ravitch versus Nuss procedure for pectus excavatum: systematic review and meta-analysis. Ann Cardiothorac Surg. 2016 Sep;5(5):409421. doi: 10.21037/acs.2016.08.06. Erratum in: Ann Cardiothorac Surg. 2016 Nov;5(6):593. https://doi.org/10.21037/ acs.2016.08.06
4. Mao YZ, Tang S, Li S. Comparison of the Nuss versus Ravitch procedure for pectus excavatum repair: an updated meta-analysis. J Pediatr Surg. 2017 Oct;52(10):1545-1552. Epub 2017 Jun 3. https://doi.org/10.1016/j.jpedsurg.2017.05.028

5. Bardají C, Cassou L. Taulinoplasty: the traction technique-a new extrathoracic repair for pectus excavatum. Ann Cardiothorac Surg. 2016 Sep;5(5):519-522. https://doi.org/10.21037/ acs.2016.09.07

6. Marcos-Ramírez ER, Garza-Cerna JA, Fonseca-Sada I, Téllez-Aguilera A, Salazar-Palafox S, Montes-Tapia F, Wong-Jaen M, MuñozMaldonado GE. Pectus up: la nueva técnica de tracción para pectus excavatum. Primera cirugía en el noreste de México. Cir Cir. 2020;88(Suppl 2):56-59. English. https://doi.org/10.24875/ CIRU.20000285

7. Raj N. Regional anesthesia for sternotomy and bypass-Beyond the epidural. Paediatr Anaesth. 2019 May;29(5):519-529. https://doi. org/10.1111/pan.13626

8. Fujii S, Bairagi R, Roche M, Zhou JR. Transversus Thoracis Muscle Plane Block. Biomed Res Int. 2019 Jul 7;2019:1716365. https:// doi.org/10.1155/2019/1716365 